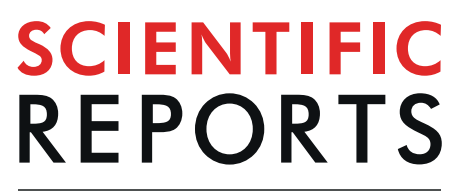

natureresearch

Check for updates

\title{
Adipose tissue in health and disease through the lens of its building blocks
}

\author{
Michael Lenz ${ }^{1,2,3 凶}$, Ilja C. W. Arts ${ }^{1,4}$, Ralf L. M. Peeters ${ }^{1,5}$, Theo M. de Kok ${ }^{1,6} \&$ \\ Gökhan Ertaylan ${ }^{1,7 凶}$
}

Understanding adipose tissue cellular heterogeneity and homeostasis is essential to comprehend the cell type dynamics in metabolic diseases. Cellular subpopulations in the adipose tissue have been related to disease development, but efforts towards characterizing the adipose tissue cell type composition are limited. Here, we identify the cell type composition of the adipose tissue by using gene expression deconvolution of large amounts of publicly available transcriptomics level data. The proposed approach allows to present a comprehensive study of adipose tissue cell type composition, determining the relative amounts of 21 different cell types in 1282 adipose tissue samples detailing differences across four adipose tissue depots, between genders, across ranges of BMI and in different stages of type-2 diabetes. We compare our results to previous marker-based studies by conducting a literature review of adipose tissue cell type composition and propose candidate cellular markers to distinguish different cell types within the adipose tissue. This analysis reveals gender-specific differences in $\mathrm{CD4}^{+}$and $\mathrm{CD}^{+} \mathrm{T}$ cell subsets; identifies adipose tissue as rich source of multipotent stem/ stromal cells; and highlights a strongly increased immune cell content in epicardial and pericardial adipose tissue compared to subcutaneous and omental depots. Overall, this systematic analysis provides comprehensive insights into adipose tissue cell-type heterogeneity in health and disease.

In multicellular organisms, a wide variety of specialized cells work in synergy, form tissues and perform essential tasks required for the functioning and survival of the organism. The homeostasis in the system is maintained also at the cellular level, such that defective, old, damaged or infected cells, or cells that are harmful to their environment either go through programmed cell death (apoptosis) or are actively detected and killed by the immune system. Hence, many molecular sensors and inter-cellular mechanisms are evolved to ensure this cellular heterogeneity is maintained at the tissue level.

Adipose tissue (AT) is no exception in this regard. It is composed of adipocytes, immune system cells, endothelial cells (blood and lymph vessels) and stem/stromal cells. Collectively, these cell types facilitate the functions associated with the tissue as an endocrine organ, energy depot, and major player in energy metabolism ${ }^{1}$. To a large extent, it consists of adipocytes, which are commonly referred to as the fat depots in the body. Furthermore, adipose tissue has the unique ability to expand and shrink in significant proportions within the same individual over time. It can account for as little as $3 \%$ of total body weight in elite athletes or as much as $70 \%$ in morbidly obese individuals ${ }^{2}$.

The conventional understanding of the adipose tissue portrays a fairly homogeneous tissue, responding to higher energy intake by expanding and to lower energy intake by shrinking. In contrast to this conventional belief, research in the last decade has focused on the different cellular subpopulations in the adipose tissue and their relation to (metabolic) health and disease ${ }^{3,4}$. This research provided deeper insights into disease development

\footnotetext{
${ }^{1}$ Maastricht Centre for Systems Biology (MaCSBio), Maastricht University, Maastricht, The Netherlands. ${ }^{2}$ Institute of Organismic and Molecular Evolution, Johannes Gutenberg University Mainz, Mainz, Germany. ${ }^{3}$ Preventive Cardiology and Preventive Medicine - Center for Cardiology, University Medical Center of the Johannes GutenbergUniversity Mainz, Mainz, Germany. ${ }^{4}$ Department of Epidemiology, CARIM School of Cardiovascular Diseases, Maastricht University, Maastricht, The Netherlands. ${ }^{5}$ Department of Data Science \& Knowledge Engineering, Maastricht University, Maastricht, The Netherlands. ${ }^{6}$ Department of Toxicogenomics, GROW School of Oncology and Developmental Biology, Maastricht University, Maastricht, The Netherlands. ${ }^{7}$ Health, Flemish Institute for Technological Research (VITO), Mol, Belgium. ${ }^{凶}$ e-mail: michael.I.lenz@gmail.com; gokhan.ertaylan@vito.be
} 


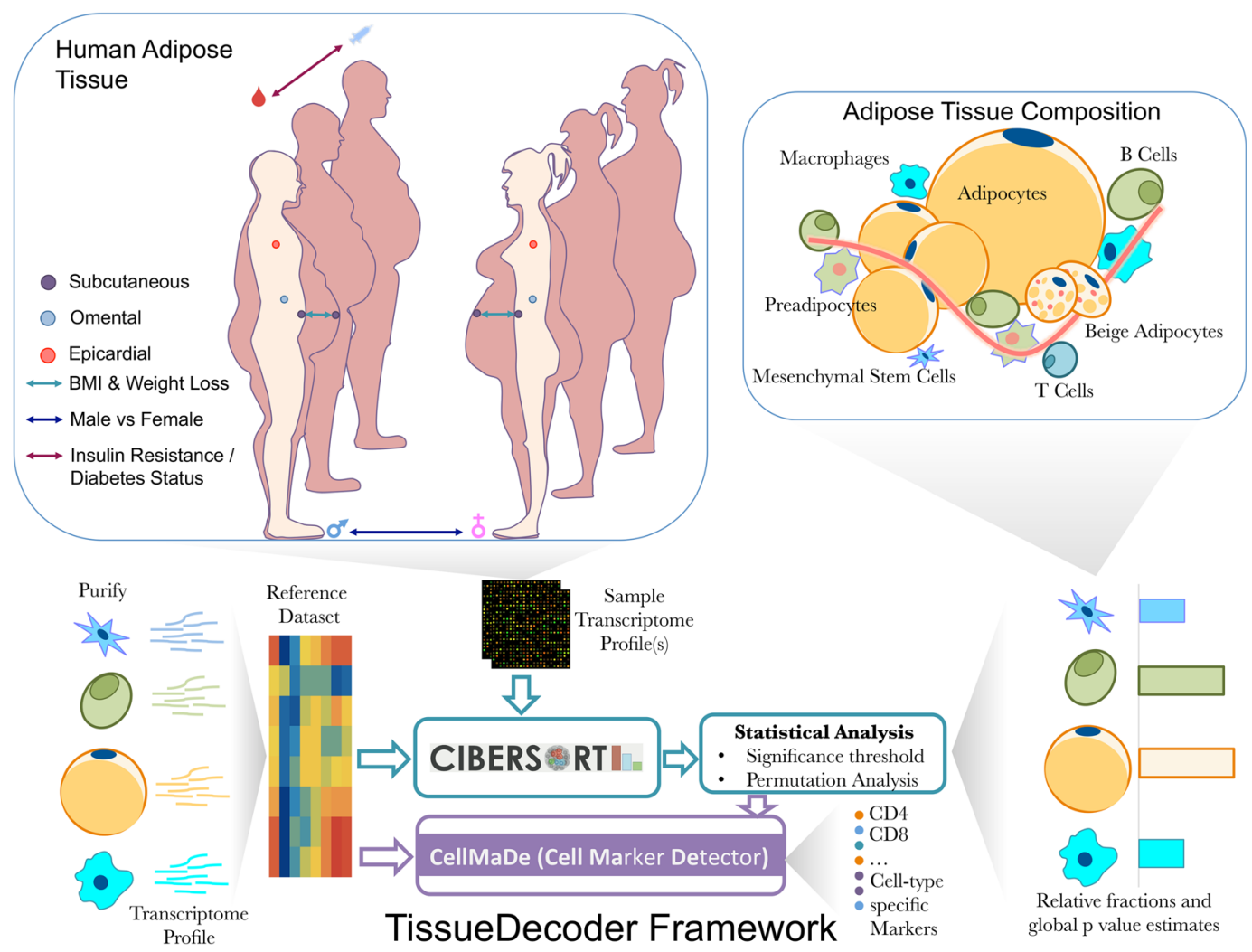

Figure 1. TissueDecoder the analysis of phenotypic traits. and the deconvolution with CIBERSORT.

and progression in complex diseases (such as heart disease or diabetes) as well as inter-individual differences in disease etiology, paving the way for improved subtyping of patients for targeted therapies.

However, so far the efforts towards characterizing the adipose tissue cell type composition are limited, partially due to lack of robust cell surface markers identifying subpopulations of cells, but also due to limited access to tissue samples and the labor-intensive process required to identify them, such as immunohistochemistry or flow cytometry. Furthermore, the markers used to define a subpopulation of cells within the adipose tissue can differ greatly across studies, impeding reproducibility and leading to discrepancies across studies. Markers are usually defined for general purpose, and not designed to be specific for a tissue, bringing in to question their specificity and sensitivity.

In this paper, we propose a novel way of determining the adipose tissue cell type composition from whole tissue microarray and RNA-Seq based gene expression profiles. Our proposed TissueDecoder framework builds upon a recently published gene expression deconvolution algorithm ${ }^{5}$, and facilitates reuse of published gene expression data for determining adipose tissue cell type composition across various depots and phenotypic traits (Fig. 1). In this way, we are able to determine the relative fraction of 21 different cell types in 1282 adipose tissue samples, presenting the most comprehensive study of adipose tissue cell type composition to date.

We determine the cell type composition of four different adipose tissue depots, including rare samples from epicardial (EAT) and pericardial adipose tissue (PAT), contextualize our results via a quantitative literature review and validate our approach using an independent dataset. Furthermore, we exemplify the usability of our approach by analyzing differences in adipose tissue cell type composition between genders, across ranges of BMI, and in different stages of type 2 diabetes development.

Finally, we demonstrate the applicability and reproducibility of our approach in RNA-Seq level data by using independent studies.

Our study highlights the heterogeneity in reported studies of adipose tissue cell type composition and contributes to a better standardization by making our curated adipose tissue-specific signature matrix (AT21) available for future studies (Supplementary Data S1).

\section{Methods}

TissueDecoder Framework. Deconvolution of adipose tissue cell types. Our approach for determining the adipose tissue cell type composition from whole-tissue gene expression data employs the CIBERSORT deconvolution algorithm ${ }^{5}$. CIBERSORT uses a reference dataset of gene expression profiles from isolated cell types to generate a signature matrix, which is subsequently used as independent variable in nu-support vector regression $(\nu$-SVR) to determine the cell type composition of whole-tissue samples. In this study, we extend the CIBERSORT signature matrix library with a novel signature matrix, termed AT21, since CIBERSORT is limited to blood due to its default signature. This broadens CIBERSORT's applicability to deconvolution of the adipose tissue. 
A

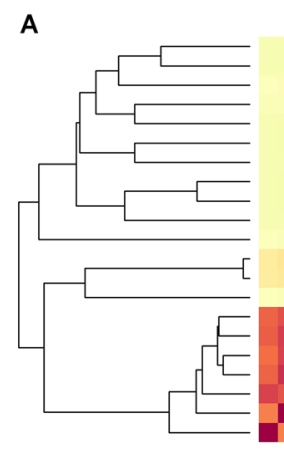

Correlations of signatures
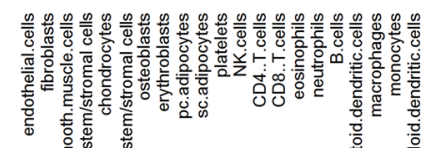
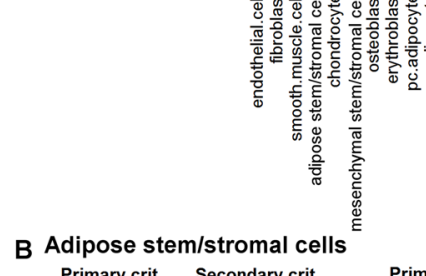

B Adipose stem/stromal cells
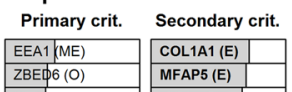

\begin{tabular}{|l}
\hline ZBED6 (O) \\
\hline Hs.5
\end{tabular}

\begin{tabular}{|l|}
\hline Hs.5 6 (6023 (O) \\
\hline Hs.
\end{tabular}

Hs.503911 (O)

ABCA9 (M)

\begin{tabular}{|l}
\hline SFRP4 (ME) \\
\hline U $80771(0)$
\end{tabular}

\begin{tabular}{|l|}
\hline U $89771(0)$ \\
\hline Hs. $113812(0)$ \\
\hline
\end{tabular}

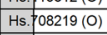

\begin{tabular}{l|l}
\hline GUEY1A2 (M) \\
\hline
\end{tabular}

CD10 [MME] (ME) $\mathrm{CD} \$ 4$
$\mathrm{C}(\mathrm{ME})$ \begin{tabular}{|l|l}
\hline CD90 [THY1] (ME) \\
\hline
\end{tabular}

\begin{tabular}{lllllllllll}
\hline 0 & 2 & 4 & 6 & 8 & 0 & 2 & 4 & 6 & 8
\end{tabular}

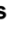

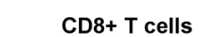

Primary crit. Secondary crit.

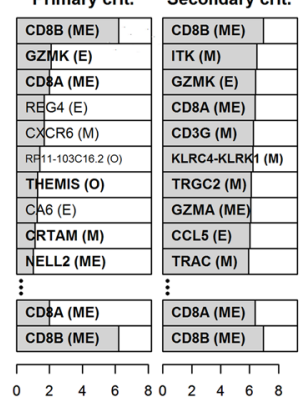

Cell types

5 GSE28490

macrophages

plasmacy

neutrophils

eosinophils

CD8..T.cells

CD4..T.ce
NK.cells

NK.cells

sc.adipocyte

pc.adipocytes

pc.adipocytes

osteoblasts

mes. stem/stromal cells

chondrocytes

adipose stem/stromal c

smooth.muscle.cel

fibroblasts

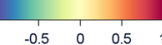

$\begin{array}{llll}-1 & -0.5 & 0 & 0.5\end{array}$

8 GSE28490

12 GSE11524

13 GSE26339

8 GSE49910

8 GSE49910

6 GSE16464

$\begin{array}{ll}3 & \text { GSE9451 } \\ 4 & \text { GSE13791 }\end{array}$
24 GSE11864, GSE22373, GSE28490, GSE49910, GSE7807 19 GSE11886, GSE7568

5 GSE28490,

9 GSE28490, GSE49910

8 GSE18810, GSE28490, GSE49910

11 GSE28490, GSE49910

8 GSE28490, GSE49910

6 GSE28490, GSE8059

6 GSE36923, GSE9451

21 GSE17090, GSE20697, GSE61302

8 GSE11367, GSE13791

Figure 2. AT21 Signature Matrix: (A) Heatmap showing the correlations of signatures for the reference dataset compiled from various studies reporting isolated cell type transcriptome profiles. The dendrogram (on the left) shows the clustering within the reference dataset. The dendrogram is constructed using hierarchical clustering with 1-correlation as the distance and average linkage as the linkage criteria. The number of samples contained for each cell type $(\mathrm{N})$, and the GEO accession numbers per study is shown on the right. Three groups revealed higher correlation and clustered together in the reference dataset in accordance with our expectations: Highest correlation is observed between subcutaneous and pericardial adipocytes followed by the cell types of the mesenchymal stem/stromal cell origin (osteoblasts, mesenchymal stem/stromal cells, chondrocytes and Adipose Stem/Stromal Cells - ASCs) and finally the T cell group (CD4 ${ }^{+} \mathrm{T}$ and $\mathrm{CD} 8^{+} \mathrm{T}$ cell subsets) follow. (B) Bar plots showing top ten cell type markers identified by the primary criterion and the secondary criterion of CellMaDe. Below each column conventional markers are demonstrated with their associated score. Bold font indicates the presence of the gene in the AT21 signature matrix and red colored bars indicate the value of the (primary/ secondary) criterion was negative. Letters in brackets (M: membrane, E: extracellular, ME: both membrane and extracellular, or O: other) specify the gene ontology cellular location of the corresponding protein.

AT21 signature matrix. We have generated the AT21 signature matrix based on publicly available data from the Affymetrix Human U133 Plus 2.0 microarray (GEO identifier 'GPL570'). We restricted our search to this microarray platform to avoid compatibility problems with cross-platform differences. However, we have also performed independent validation using Affymetrix Human Transcriptome Array 2.0 and RNA-Seq level data.

For our AT21 signature matrix, 21 cell types (Fig. 2A) from four cellular archetypes, namely immune cells (12 cell types), stem/stromal cells (adipose and (bone marrow-derived) mesenchymal), adipocytes (from subcutaneous adipose tissue (SAT) and PAT), and other cell types (endothelial cells, fibroblasts, smooth muscle cells, chondrocytes, and osteoblasts) were selected. In the remainder of this manuscript, we refer to adipose stem/ stromal cells (ASCs) and to mesenchymal stem/stromal cells (MSCs) as two distinct cell subpopulations. The clear distinction between ASCs with respect to the bone-marrow-derived MSCs included in this study is by their positivity for CD36 and negativity for CD106 as described by the International Fat Applied Technology Society

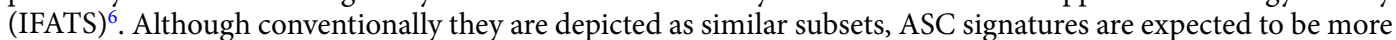
profoundly associated with adipose tissue presence than MSCs.

We downloaded a total of 204 Affymetrix microarray datasets (Supplementary Data S2), containing samples from all 21 isolated cell types, from the gene expression omnibus (GEO) database ${ }^{7}$, jointly preprocessed them, and generated the AT2 1 signature matrix using the CIBERSORT online service ${ }^{5}$ (see Supplementary Methods), which is provided as Supplementary Data S1. 
AT4 signature matrix with cell types isolated from adipose tissue. One potential caveat and limitation of the AT21 signature matrix is the partial use of reference cell types that were not directly isolated from adipose tissue, as such data was (for some cell types) not available in public databases. Therefore, we generated an alternative reference dataset containing solely cell types that were directly isolated from adipose tissue for evaluation and comparison to the results obtained using AT21. The resulting AT4 signature matrix is limited to four cell fractions (adipocytes, stem/stromal cells, monocytes/macrophages, and other leukocytes) available in dataset GSE80654, which utilized the Affymetrix Human Transcriptome Array 2.0. After composition of the reference dataset, AT4 was generated in the same way as AT21 (see Supplementary Methods) and is provided as Supplementary Data S6.

Adipose tissue samples used for deconvolution. We first utilized the AT21 signature matrix and the CIBERSORT online tool to determine the cell type composition of 779 adipose tissue samples hybridized to the same microarray platform as the reference dataset (Affymetrix Human U133 Plus 2.0). The adipose tissue samples came from four different adipose depots (subcutaneous, $\mathrm{n}=616$; omental, $\mathrm{n}=51$; pericardial, $\mathrm{n}=66$; epicardial, $\mathrm{n}=46$ ) and 12 original studies (Supplementary Methods, Supplementary Data S2).

Similarly, the deconvolution approach was applied to the isolated cell types from the AT21 reference dataset, to check how well closely related cell types (e.g. subcutaneous and pericardial adipocytes) can be distinguished.

Next to this, an independent cross-platform validation of the TissueDecoder framework was conducted on a "validation dataset" by deconvolving samples from eight isolated cell types hybridized to the Affymetrix Human Transcriptome Array 2.0 (Supplementary Methods, Supplementary Data S2, Supplementary Fig. S1).

Furthermore, we tested the compatibility of our approach with the RNA-Seq technology (Supplementary Methods) by deconvolving 503 adipose tissue samples from 5 original studies using the AT21 signature matrix with CIBERSORT. In combination with the 779 adipose tissue samples described above, this resulted in a total of 1282 adipose tissue samples that were deconvoluted with AT21 and evaluated regarding their mutual agreement and consistency with literature.

The AT4 signature matrix was utilized to deconvolve (i) the six stromal vascular fraction samples from dataset GSE80654 and (ii) the 779 adipose tissue samples (Affymetrix Human U133 Plus 2.0) that we analyzed with AT21 before.

Cell type-specific marker detector (CellMaDe). We have developed the Cell Marker Detector (CellMaDe) that uses two criteria to pinpoint i) highly specific markers that are only expressed in the target cell type and not in any other cell type of the tissue, referred to as primary markers (Eq. 1 below), and ii) markers expressed in the target cell type that might also be expressed in some other cell types, referred to as secondary markers (Eq. 2 below). A classical approach to cell type identification is the use of antibodies for specific marker proteins in immunohistochemistry or flow cytometry-based approaches. For these approaches, it is usually necessary to know cell type-specific markers that are not expressed (or only much lower expressed) in any of the other cell types, i.e. 'primary markers. This approach comes with the limitation that some cell types are difficult to distinguish based on the expression of single marker proteins. For instance, mesenchymal stem/stromal cells are typically characterized by a combination of several markers as well as functional assays ${ }^{8}$. Thus, where primary markers are not applicable, the idea is to combine several 'secondary markers' to receive unambiguous cell type identification.

In CellMaDe, we define the 'primary criterion' and the 'secondary criterion' to determine primary and secondary markers, respectively, as follows:

For each gene and each cell type, the primary criterion is calculated as the average expression of that gene in this cell type, minus the largest average expression of that gene in any other cell type, i.e.

$$
\text { crit }_{\text {primary }}(i, j)=g_{i j}^{\prime}-\max _{k \neq j}\left(g_{i k}^{\prime}\right)
$$

where $g^{\prime}{ }_{i j}$ is the average expression of gene $i$ in cell type $j$. The secondary criterion is calculated for each gene and each cell type as the average expression of that gene in this cell type minus the average expression of that gene in all other cell types, i.e.

$$
\text { crit }_{\text {secondary }}(i, j)=g_{i j}^{\prime}-\operatorname{mean}_{k \neq j}\left(g_{i k}^{\prime}\right)
$$

This results in a primary criterion score and a secondary criterion score for each gene which are then used to obtain the ranked lists for each score (Supplementary Fig. S2).

The validity of the most promising primary marker identified per cell type was further evaluated based on a large compendium of 394 anatomically annotated tissue expression profiles using Genevestigator (see Supplementary Methods, Supplementary Fig. S3).

We have also annotated the top ten primary markers and selected conventional markers with their corresponding Gene Ontology (GO) cellular locations ${ }^{9}$ (Fig. 2B). Since these markers are identified to be used potentially in cell sorting, identification, or tracking applications, they are usually desired to be either on the cell surface, membrane or on the extracellular part of the membrane. Hence, we have used four different notations depicting the locations of interest (M: membrane, E: extracellular, ME: both membrane and extracellular, or O: other) for the corresponding proteins (Fig. 2B, Supplementary Fig. S2).

Literature review of adipose tissue cell type composition. We performed a literature review of quantitative reports about the cellular composition of the human adipose tissue for comparison with our results. Using a manual (non-systematic) search, we have extracted original and review articles, which we then further examined for relevant referenced studies. We selected a total of 25 original studies (Supplementary Data S4) that report cell 
type-specific cell counts of non-adipocytes in human adipose tissue (SAT and/or Omental Adipose Tissue; OAT), from which we extracted information about the cell type, number of people included with information on gender and BMI, adipose tissue depot, biopsy type, method and marker used for cell counting, as well as mean, standard deviation/standard error, minimum and maximum of reported counts (where available). In many studies, cell counts were only reported graphically, in which cases we extracted them manually through visual inspection.

Eighteen out of the twenty-five studies reported macrophage counts, of which eleven exclusively focused on macrophages. Nine studies reported ASC fractions; frequencies of CD4 ${ }^{+} \mathrm{T}$ cells, $\mathrm{CD} 8^{+} \mathrm{T}$ cells, and endothelial cells were reported in five studies each and those of B cells in 4 studies. Natural killer cell frequencies were reported in two studies and frequencies of myeloid dendritic cells, and fibroblasts were mentioned only in one study each. We have made the effort to compile the literature to the best of our knowledge, however, our list may not be exhaustive since several studies that focus on the isolation (rather than quantification) of adipose stromal cells also report cell numbers. We did not find any cell counts for the remaining eleven cell types in our signature matrix (monocytes, plasmacytoid dendritic cells, eosinophils, neutrophils, platelets, erythroblasts, smooth muscle cells, chondrocytes, osteoblasts, mesenchymal stem/stromal cells, and pericardial adipocytes).

Direct comparison of the different studies is complicated not only by heterogeneity in the methods and markers used for cell counting but also by the heterogeneity in the units in which cell counts were expressed. The 25 studies used a total of six different units, namely (i) number per 100 adipocytes, (ii) number per g of adipose tissue, (iii) percent of SVF cells, (iv) number per total number of nuclei, (v) number per high power field, and (vi) number per $\mathrm{mm}^{2}$. We converted all units into percent of total cells using the formulas described in the Supplementary Methods, excluding the two studies expressing the cell numbers in number per high power field and number per $\mathrm{mm}^{2}$.

Statistics. Non-parametric statistical tests were utilized to associate changes in cell type composition with available phenotypic information in the public datasets, as the relative cell type composition frequently had a skewed distribution and its range of possible values is limited between 0 and 1 . Repeated measures within the same person (i.e. intervention studies with placebo, resveratrol, or calorie restriction) as well as the twin study (heavier vs. leaner twin concordant, heavier vs. leaner twin discordant) were considered as paired samples and analyzed using Wilcoxon signed rank test. Non-paired comparisons were performed using Wilcoxon rank sum test. Continuous analyses testing the association of age and BMI with adipose tissue cell type composition were performed using permutation tests based on Spearman correlation ('cor.test' function of package 'stats' in R). We report significant results both with and without Benjamini-Hochberg correction for multiple testing for the 336 comparisons (21 cell types times 16 phenotypic traits). Reported p-values are without correction.

Ethics approval and consent to participate. Not applicable. All the studies included in this work are from earlier published work; hence the ethical approval for each study can be found in publications accompanying them.

Consent for publication. An earlier version of this article is present on bioRvix repository website and can be accessed on http://https://www.biorxiv.org/content/10.1101/316083v1. This article is not published nor is under publication elsewhere.

\section{Results}

Gene expression-based deconvolution of adipose tissue samples using the AT21 signature matrix. The AT21 signature matrix was developed with the aim to determine the cellular composition of adipose tissue samples. It consists of cell type-specific expression profiles of 1872 microarray probes from 21 cell types. The 1872 probes were selected to optimize the ability of the signature matrix to distinguish between different cell types, which is reflected by minimizing the information overlap between cell types (implemented via a minimization of the condition number, see Supplementary Methods).

In Fig. $2 \mathrm{~A}$ we show the correlations of signatures between the different cell types, revealing high correlations between related cell types, such as subcutaneous and pericardial adipocytes, or mesenchymal stem/stromal cells, chondrocytes, osteoblasts, and adipose stem/stromal cells (ASCs). In light of these high correlations between some cell types, we set out to investigate the ability of our approach to distinguish between similar cell types by the following two strategies. First, we apply the deconvolution approach to the reference dataset itself, resulting in a clear distinction between cell types (Fig. 3A) as a positive control, to demonstrate that the method proposed can indeed identify pure cell signatures from the reference dataset clearly. We also show in the same figure (Fig. 3B,C) the normalized expression of conventional markers and CellMaDe predicted primary markers for comparison from the cell types in the reference dataset. The figure shows the comparison in terms of cell type specific normalized expression of conventional markers vs CellMaDe predicted markers for each cell type. Second, we perform deconvolution analysis with a set of 779 adipose tissue samples (Supplementary Data S2) from four different adipose tissue depots (SAT, OAT, PAT, and EAT) and check how well the results agree with the body of literature. This second analysis shows that the adipose tissue samples are predicted to have on average $14.5 \%$ ASCs, while the sum of the three related cell types (mesenchymal stem/stromal cells, osteoblasts, and chondrocytes) is below $1 \%$ on average, revealing our ability to correctly distinguish between these cell types (Supplementary Fig. S4). Furthermore, $95 \%$ of the SAT samples have a pericardial adipocyte score of $0 \%$ and an average subcutaneous adipocyte score of $74 \%$, whereas OAT, EAT, and PAT samples are mostly predicted to have more pericardial adipocytes than subcutaneous adipocytes, although the distinction is less clear. This reveals the clear difference between subcutaneous adipocytes and adipocytes from other adipose depots in proximity to internal organs, which can be detected by the proposed methodology. 


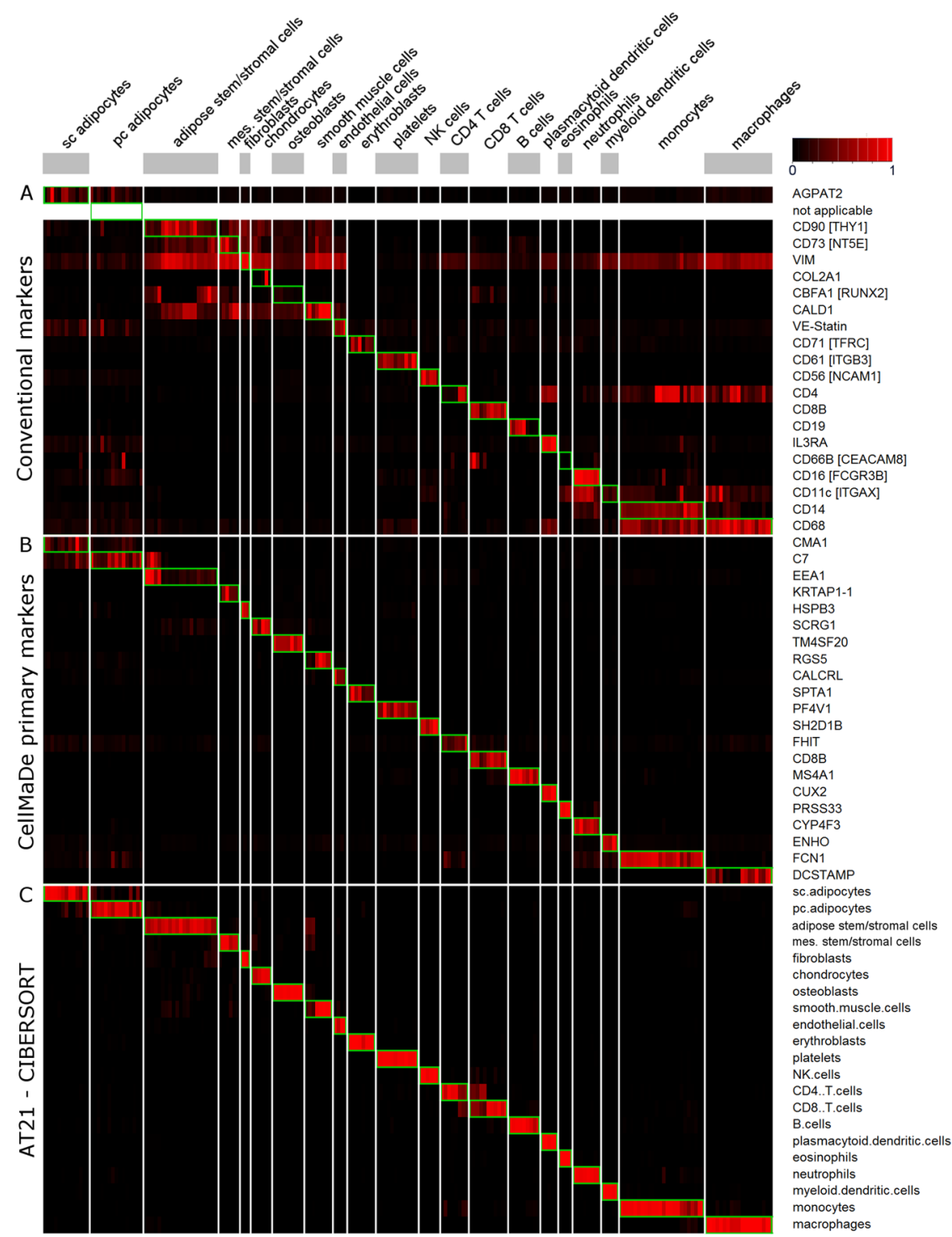

Figure 3. Cell Type Specificity of TissueDecoder: Heatmaps of AT21 signature matrix showing (A) cell type composition prediction from CIBERSORT with AT21 signature matrix, (B) the normalized expression of selected conventional markers from literature and $(\mathbf{C})$ the normalized expression of CellMaDe predicted primary markers. The common xaxis above the figure denotes the samples used for annotating cell types from the AT21 signature matrix. CellMaDe and CIBERSORT are both trained with AT21 signature matrix, therefore $(\mathbf{A}, \mathbf{C})$ are optimal results for both techniques whereas (B) represents the separation power of conventional markers on the AT21 signature matrix. Green boxes indicate for each cell type (columns) the row with its corresponding marker.

Next to these evaluations, we utilized an independent cross-platform (different Affymetrix microarray platforms) validation dataset to test the CIBERSORT-based deconvolution with the AT21 signature matrix, showing that our approach, correctly provides highest percentages for the respective isolated cell type for all tested cell types in the validation dataset (Supplementary Fig. S1C).

The mean estimated percentages of the isolated cell type are $69.2 \%$ for subcutaneous adipocytes, $57.1 \%$ for ASCs, $89.9 \%$ for B cells, $84.8 \%$ for CD4 ${ }^{+} \mathrm{T}$ cells, $71.0 \%$ for $\mathrm{CD}^{+} \mathrm{T}$ cells, $62.0 \%$ for $\mathrm{NK}$ cells, and $90.5 \%$ for monocytes. The $\mathrm{CD} 14^{+}$fraction of the adipose tissue (monocytes/macrophages) is predicted to consist mainly of macrophages (25.4\%), myeloid dendritic cells (24.4\%) and monocytes (18.1\%). The deviation from the optimal results based on the reference data itself (Fig. 3A) can be explained by cross-platform differences between the validation and reference datasets (the validation dataset and reference dataset have been hybridized to two different Affymetrix microarrays).

CellMaDe primary and secondary markers. Next, we further characterize the signature matrix by investigating which genes are incorporated in it, and by comparing their cell type specificity to that of conventionally used cell type markers using the primary and secondary marker criteria (Eqs. 1 and 2 in methods section). This 
has resulted in a ranking of all the probes present on the array according to their primary and secondary criterion scores. In Fig. 2B, we show the top 10 genes having the highest primary and secondary criterion score from four cell types - namely ASCs, CD8 ${ }^{+} \mathrm{T}$ cells, macrophages and subcutaneous adipocytes - and indicate their cellular location according to gene ontology terms (for all 21 cell types see Supplementary Fig. S2, Supplementary Data S3). All genes that are present in the AT21 signature matrix are marked in bold, showing that CIBERSORT algorithm mainly selects a combination of secondary markers (CIBERSORT's gene selection criterion is comparable to our secondary marker criterion), while primary markers and conventional markers are not always included in the signature matrix.

This is in contrast to classical marker-based approaches such as immunohistochemistry, which rely heavily on the cell type specificity of a single (primary) marker. Nevertheless, conventional markers such as CD68 for macrophages are also expressed in other cell types, such as monocytes, plasmacytoid dendritic cells ${ }^{10}$, and to a lesser degree also in fibroblasts and endothelial cells ${ }^{11,12}$, which is also indicated by its relatively low primary criterion score (Fig. 2B). We, therefore, propose the primary markers identified by CellMaDe to be further confirmed experimentally before clarified as alternative markers for the cell types within the adipose tissue.

For $\mathrm{CD}^{+} \mathrm{T}$ cells, the conventionally used marker (Cluster of differentiation 8B; CD8B) is identified as the strongest primary marker, which was not very surprising. However, Cluster of differentiation 4 (CD4) is not very specific for $\mathrm{CD}^{+} \mathrm{T}$ cells, as shown by its prevalent expression in other hematopoietic cell types such as monocytes or macrophages (Fig. 3B, Supplementary Fig. S2). This is also the reason that in flow cytometry, CD4 is used only after the identification of $\mathrm{CD}^{+}$cell population (T cells) to identify $\mathrm{CD} 4^{+} \mathrm{T}$ cells. The Dendrocyte Expressed Seven Transmembrane Protein (DCSTAMP) is identified as a very specific primary marker for macrophages according to our analysis. In Fig. 3C we observe that the high expression of DCSTAMP is limited to a subset of the macrophage samples, which might highlight its specificity to a subset of macrophages, e.g. macrophage giant like cells, as suggested by earlier studies ${ }^{13}$. For ASCs the identified primary marker EEA1 is not very striking. For this cell type, we suggest using a combination of secondary markers as implemented in flow cytometry gating strategies as well as in CIBERSORT algorithm.

The case of subcutaneous adipocytes seems to be similar as the primary marker CMA1 is also not very striking. This can however be attributed to the fact that pericardial adipocytes are also part of the reference matrix. Hence the primary criterion does in this case not only focus on distinguishing adipocytes from other cell types, but also on distinguishing adipocytes from two different depots. Therefore, we included an additional analysis by merging all adipocytes in the reference matrix together to determine primary markers for adipocytes. The top three primary markers identified for adipocytes in this analysis were SPARCL1, ADIPOQ, and THRSP.

For further evaluation of the identified top primary markers, we compared their expression in a more extensive independent dataset comprised of 394 anatomically annotated tissue expression profiles (Supplementary Fig. S3, Supplementary Methods). Six of the 21 primary markers are fully validated, defined as showing the highest expression in the proposed cell type, namely CALCRL for endothelial cells, SPTA1 for erythroblasts, CD8B for CD8 ${ }^{+}$T cells, MS4A1 for B cells, PRSS33 for eosinophils, and DCSTAMP for macrophages. Twelve markers are partially validated, identified as being among the top five of the 394 anatomically annotated tissue expression profiles or being expressed at a higher level only in cell types that are not related to adipose tissue, such as myocytes or neurons. Only three markers were not validated, namely EEA1 for ASCs, RGS5 for smooth muscle cells, and PF4V1 for platelets, the latter due to the absence of platelets in the validation dataset for PF4V1, preventing its proper evaluation.

Finally, we utilized the independent validation dataset to evaluate the discriminatory power of the identified top primary markers. The results of this analysis are presented in Supplementary Fig. S1 and Supplementary Data S2, showing that the primary markers are expressed and able to distinguish between cell types in the validation dataset, with the exception of CMA1 for subcutaneous adipocytes and EEA1 for ASCs (Supplementary Fig. S1B).

Contextualization of findings - a literature review. As the next step of evaluation, we have compiled quantitative literature reports of human adipose tissue cell type composition to compare the estimates of our deconvolution approach for 1119 SAT samples (616 microarray and 503 RNA-Seq datasets) and 51 OAT samples to the reported percentages in the literature.

First, we searched for any reports in the literature quantifying the percentage of adipocytes in the adipose tissue. This search, revealed a range of estimates from $\sim 93 \%{ }^{14}, \sim 70 \%{ }^{15,16}$ and $\sim 15 \%{ }^{17}$, which can potentially be explained by differences in sample processing (e.g. removal of blood vessels) and counting methods (e.g. histology vs. cell isolation, different markers). Very recently, Glastonbury and colleagues estimated the adipocyte fraction in two population-level subcutaneous adipose-tissue RNA-Seq datasets (TwinsUK, $n=766$ and the Genotype-Tissue Expression project [GTEx], $\mathrm{n}=326$ ) by estimating the relative proportions of four distinct cell types (adipocytes, macrophages, CD4 + T cells, and micro-vascular endothelial cells). Their estimation for the median percent of adipocytes is $62 \%$ for GTEx and $82 \%$ TwinsUK study ${ }^{18}$.

Subsequently, we concentrated on studies determining fractions of non-adipocytes ${ }^{19}$ and included 25 original studies in our review. The quantitative results of cell fractions extracted from these studies are presented in Fig. 4 and Supplementary Data S4 (see Supplementary Methods for methodological details). Reported cell counts for macrophages vary greatly, ranging from average counts of less than $1 \%$ of total cells in some studies up to an average of $27 \%$ of total cells in another study. These rather large differences between the studies can arise due to several factors, including (i) actual biological differences between the analyzed samples, (ii) local enrichment of macrophages (e.g. in crown-like structures) that specifically influence results with low total cell counts like immunohistochemistry, (iii) technical differences between the utilized methodologies and markers, and (iv) differences in sample handling and analysis protocols (e.g. fluorescence cutoffs, utilized antibodies). It is important to note that specifically, some immunohistochemistry studies report very high macrophage numbers (Fig. 4A). 
A

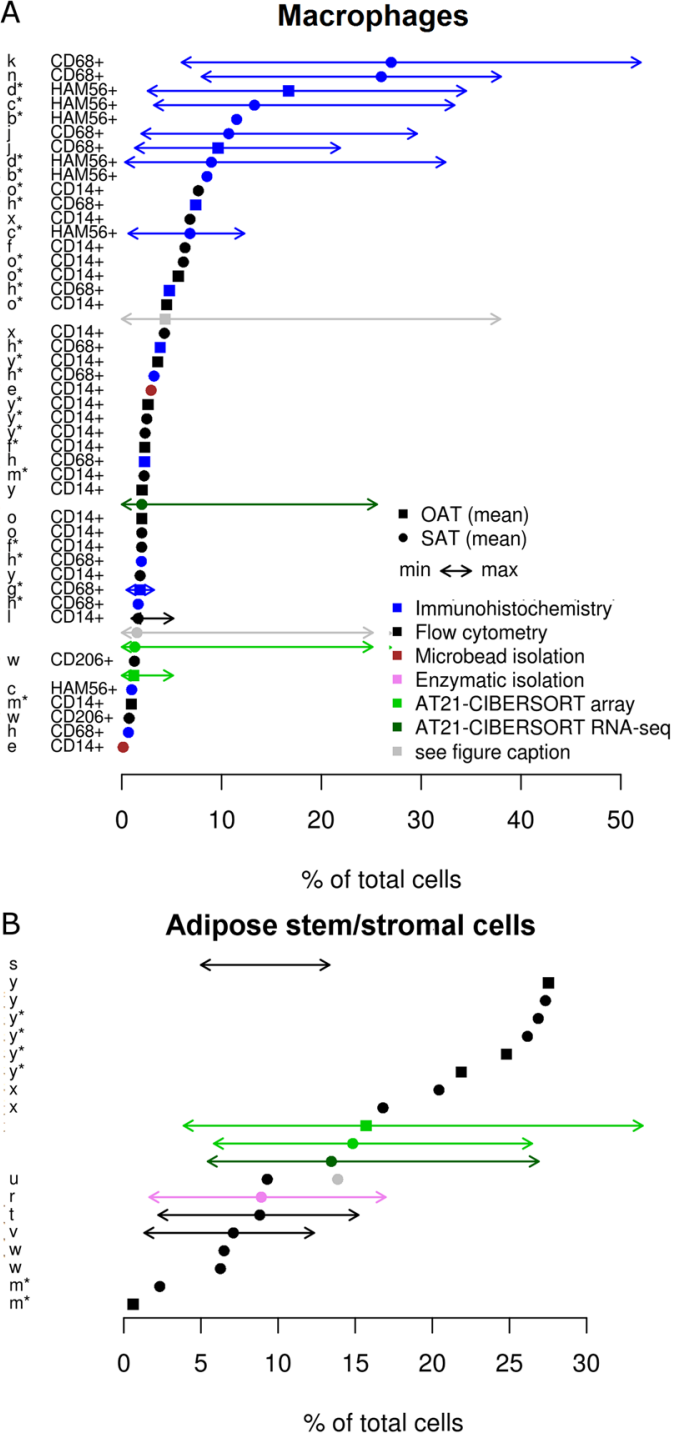

C
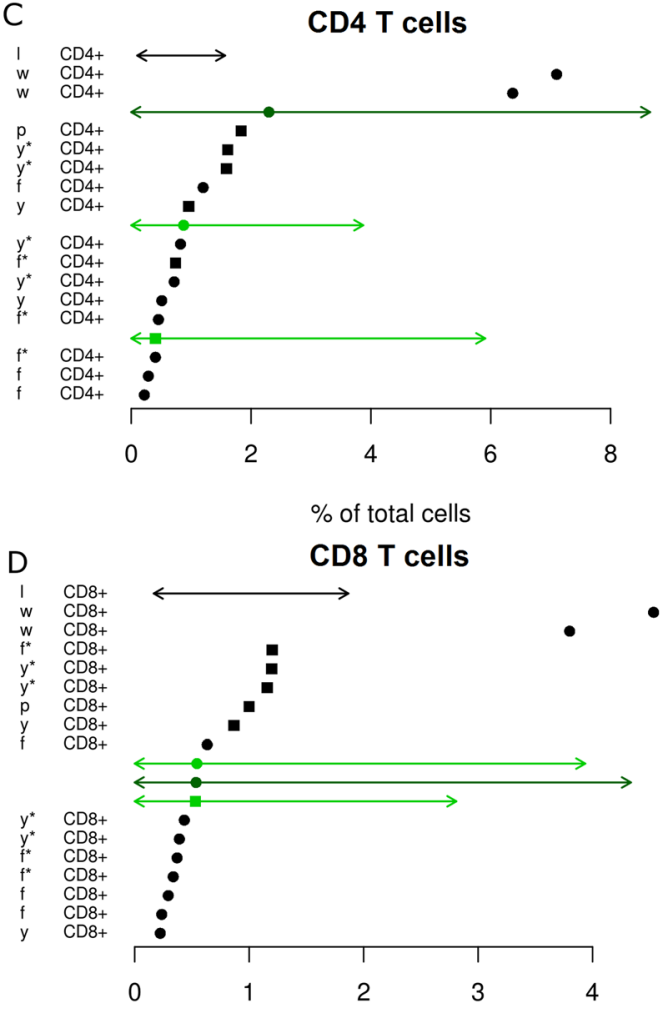

$\%$ of total cells

E

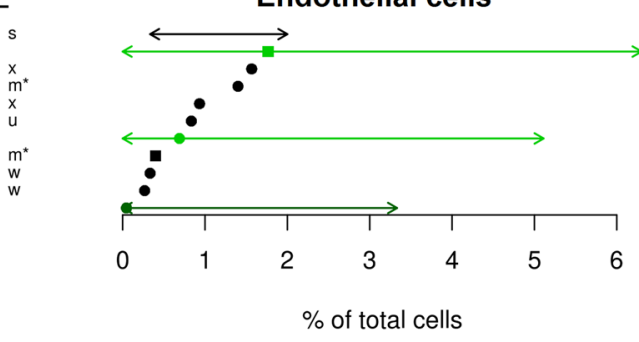

Figure 4. Review of Adipose Tissue Cellular Composition: Literature review of reported adipose tissue cellular composition in comparison to our results using CIBERSORT (in green). Shown is the mean (dots), minimum, and maximum (arrows) percentage of total cells (calculated according to assumptions and formulas stated in the supplementary methods) for five different cell types - macrophages (A), ASCs (B), CD4 ${ }^{+}$T cells $(\mathbf{C}), \mathrm{CD}^{+}$ T cells (D), and endothelial cells (E). The color specifies the method used for cell counting as indicated in the legend. The gray dots and arrows in (A) are our results where the macrophage score and monocyte score have been added together. It is shown as a comparison, since the macrophage markers CD68, HAM56, and CD14 also stain monocytes. The gray dot in (B) represents the sum of 'supra adventitial-adipose stromal cells' (black dot in the same row) and 'endothelial progenitor cells', which were distinguished in the respective study, but are likely both covered in the 'adipose stem/stromal cell' score from our AT21 signature matrix. On the left hand side of each plot the references to the studies from which the results were taken (see Supplementary Data S4) and the utilized markers are indicated. For ASCs and endothelial cells a combination of markers was used (see Supplementary Data S4). A star attached to the study reference letter indicates that the study participant had an average body mass index above 35 . It is included in the figure since it has been reported that the macrophage frequency is increased in people with severe obesity.

In addition, there may be some differences due to reported units across different studies, as the two studies with highest macrophage percentages (averages of $27 \%$ and $26 \%$ macrophages) are the only ones reporting in 'macrophages per total number of nuclei'. We have shown previously that immunohistochemistry studies from tissue slices can be biased due to reliance on observations from cross-sections (thin tissue slices) ${ }^{20}$. Therefore, it can be argued that specifically for adipocytes the cross-section may cover parts of the lipid droplet, but not the nucleus, resulting in systematic differences between counting methodologies.

In order to evaluate the potential influence of biological differences between study participants, especially with respect to their obesity status, we marked all studies involving people with an average body mass index above 35 (severe obesity) with a star (Fig. 4A). The relationship between obesity status and macrophage counts has been 
studied in several articles, reporting increased macrophage counts with increasing obesity in some, but not all studies $^{21-26}$. Nevertheless, the obesity status cannot explain the observed diversity between reported macrophage percentages in our literature review (Fig. 4A).

For comparison, we assessed inter-study differences in our analyses (only SAT), showing relatively stable results, which indicates a better standardization despite biological differences of study participants and potential differences in sample handling between different labs (Supplementary Fig. S5). Even using RNA-Seq level data to perform deconvolution resulted in a lower variance than the reported studies from the literature (Fig. 4, Supplementary Fig. S6).

In comparison to the literature reports, the estimated amount of macrophages from our analysis is at the lower end of the spectrum, with an average of $1.3 \%$ of total cells for the 616 SAT samples (median of $0.8 \%$, IQR: $0.03 \%$ $1.8 \%$ ) and $1.2 \%$ of total cells for the 51 OAT samples (median of $1.2 \%$, IQR: $0.4 \%-1.8 \%$ ). Looking at the extremes, our results confirm that there is a large range of macrophage composition with up to $25 \%$ macrophages in very rare cases.

In order to account for the potential influence of monocytes, which also express the markers utilized in the literature studies (CD14, CD68, and HAM56), we also report the combined fractions of macrophages and monocytes from our AT21-CIBERSORT approach. This amounts to an average of 1.5\% macrophages/monocytes in SAT samples and $4.3 \%$ macrophages/monocytes in OAT samples, which brings our estimates in close proximity to the mean values reported in the literature.

The amount of ASCs (mean of $14.8 \%$ in SAT and $15.7 \%$ in OAT), CD4 ${ }^{+}$T cells (mean of $0.9 \%$ in SAT and $0.4 \%$ in OAT), $\mathrm{CD}^{+} \mathrm{T}$ cells (mean of $0.5 \%$ both in SAT and OAT) and endothelial cells (mean of $0.7 \%$ in SAT and $1.8 \%$ in OAT) estimated by our approach is well in line with the literature reports (Fig. 4B-E). Similar to macrophages, literature reports of ASC quantities show large variations (Fig. 4B). We have identified three reasons explaining this variation. First, three of the literature studies (studies s, t, and v - see Supplementary Data S4) use adipose tissue from liposuction aspirates, which is contaminated with blood ${ }^{17}$, resulting in lower relative fractions of ASCs in the SVF. Second, some studies distinguish between endothelial progenitors and supra-adventitial adipose stem cells (e.g. studies u and v), whereas others (including our study) don't, possibly counting both subpopulations as ASCs. Notably, when adding up the two subpopulations in the study of Zimmerlin ${ }^{27}$, the resulting average fraction of 13.9\% (gray dot in Fig. 4B) is close to our estimated results. Third, the total cell yield and stem/stromal cell percentage vary widely depending on the method used for isolation of SVF ${ }^{28,29}$, which can potentially explain the very low numbers reported by Viardot and colleagues ${ }^{30}$ (study $\mathrm{m}$ ).

There are several cell types such as plasmacytoid dendritic cells, neutrophils, or eosinophils for which we could not find any results in the literature and thus we are reporting their frequency in the human adipose tissue for the first time. Some cell types such as platelets and erythroblasts are mainly included as a control in the AT21 signature matrix, allowing for identification of the presence of blood within the samples.

Reference dataset with cell types isolated from adipose tissue. For further verification of the AT21 deconvolution results, we compared them to the deconvolution based on the AT4 signature matrix consisting of four cell fractions isolated from adipose tissue.

In order to rule out differences in study population and tissue sampling procedures (while keeping differences in cell type granularity, reference samples, and performing cross-platform analysis) we used the ex-vivo reference to deconvolve the 779 adipose tissue samples from Affymetrix Human U133 Plus 2.0 array that we analyzed with our AT21 signature matrix before. The resulting cell percentages (Supplementary Fig. S7) are in a similar range as the results obtained using AT21 as reference (although monocyte/macrophage percentages are a bit higher) and correlate reasonably well with them, revealing Spearman and Pearson correlations between 0.41 and 0.87 (Supplementary Fig. S8). Nevertheless, our analysis demonstrates that choice of cell types and their origin can have potential impact on the level of detail in the results although the overall distribution is conserved.

For further evaluation of our deconvolution approach, we used this 'ex-vivo reference' to deconvolve samples consisting of the stromal vascular fraction of adipose tissue (also from dataset GSE80654), revealing a cell type distribution of $53 \%$ stem/stromal cells, $27 \%$ monocytes/macrophages, $19 \%$ other leukocytes, and $1 \%$ adipocytes on average (see Supplementary Fig. S9) from $n=6$ individuals out of a total of $n=10$. The data for the remaining four individuals was not available. The flow cytometry results reported slightly different averages of $62 \%$ stem/stromal cells, $13 \%$ monocytes/macrophages, $12 \%$ other leukocytes, $3 \%$ endothelial cells, $~ 10 \%$ unspecified), despite coming from the larger sample size of $n=10$ individuals in the original study ${ }^{31}$. Both results confirm the high amount of stem/stromal cells in adipose tissue and (after unit conversion from cells in SVF to cells in adipose tissue - see methods) are reasonably similar to our average results applying AT21 to adipose tissue, when considering the differences in study population, adipose tissue sampling methodologies, and granularity of cell type distinction ( 4 vs. 21 cell types).

Comparison of four adipose tissue depots. Next, we compare the cell type composition of four adipose tissue depots (SAT, OAT, PAT, and EAT) by reporting their average cell type composition (Fig. 5, detailed in Supplementary Fig. S4). This indicates that SAT has the highest percentage of adipocytes (74\%) followed by OAT (66.4\%), EAT (59.5\%) and PAT (59.4\%), while EAT and PAT have far more immune cells (20.8\% and $20.9 \%$, respectively) compared to OAT $(9.8 \%)$ and SAT (7.4\%). Furthermore, OAT is the richest source of stem/stromal cells (17.2\% compared to $14.9 \%$ for SAT, $14.1 \%$ for EAT and $12.4 \%$ for PAT).

These results need to be interpreted with care due to differences in number and characteristics of people from which the samples were collected. Access to EAT and PAT is severely limited due to their physiological location and the invasive nature of the sampling procedure. Therefore PAT samples were taken from 66 patients (age: $66 \pm 8$ years) with coronary artery disease $(\mathrm{CAD})^{32}$ and the EAT samples were taken from 11 neonates $(6$ 


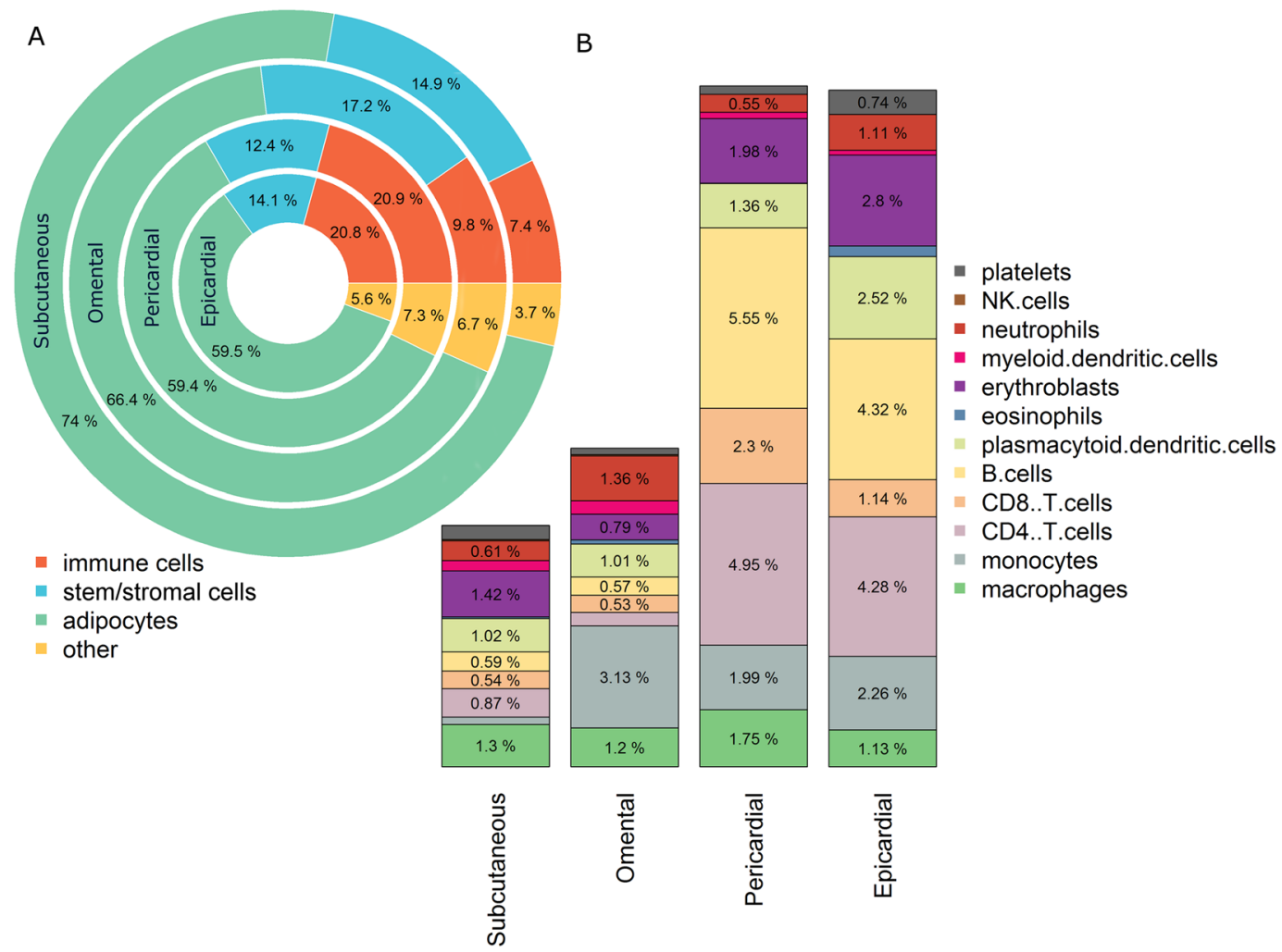

Figure 5. Estimated Percentage of Cell Types per Adipose Depot: (A) Pie chart showing the overall cell type distribution of various adipose tissue depots (Subcutaneous $-\mathrm{n}=616$, Omental $-\mathrm{n}=51$, Pericardial $-\mathrm{n}=66$, and Epicardial $-\mathrm{n}=46$ ) in terms of four main archetypes of cells in adipose tissue (Immune cells, Stem/stromal cells, Adipocytes, and other). (B) Bar plots showing the detailed distribution of the immune cell compartment from each adipose tissue depot. All values are averages of the analyzed samples from the respective depot.

to 24 days old), 28 infants (40 days to 1 year-old) and 7 children (2 to 7 years old) with congenital heart disease $(\mathrm{CHD})^{33}$.

Despite differences in the age of EAT and PAT donors, the composition of the immune cell archetype in EAT and PAT is remarkably similar to each other while being very different from SAT and OAT samples. This indicates the robustness of our results as well as the conserved nature of the cell type composition in these two adipose tissue depots surrounding the heart.

Furthermore, we report the fractions of classically designated "adaptive" immune cells, including B cells, $\mathrm{CD}^{+}$and $\mathrm{CD}^{+} \mathrm{T}$ cells, infiltrated into EAT and PAT. These adaptive immune cells are enriched in EAT and PAT, (higher in PAT than in EAT) compared to SAT and OAT depots, which could likely be due to the origin of the analyzed samples from patients with CAD or $\mathrm{CHD}$, as reported earlier for $\mathrm{CD}^{+} \mathrm{T}$ cells ${ }^{34}$. Our finding is also supported by Mazurek and colleagues ${ }^{35}$, who compared the expression of cytokines in both EAT and SAT in patients undergoing coronary artery bypass graft and found that EAT is a source of several inflammatory mediators.

A more detailed comparison of SAT and OAT was performed on the study of Hardy et al. 2011 (dataset GSE20950), in which both depots were available from the same individuals ${ }^{25}$. This analysis revealed an increased neutrophil content in SAT (also after correcting for multiple testing) and increased mesenchymal stem/stromal cell and smooth muscle cell content in OAT (Fig. 6A). Investigation of CellMaDe-derived markers for neutrophils (CYP4F3) as well as for pericardial adipocytes (C7) and subcutaneous adipocytes (CMA1) confirmed the significant difference between SAT and OAT in these cell types (also after adjustment for multiple testing).

Cell type composition across phenotypic traits. Finally, we compared the adipose tissue cell type composition between individuals with different phenotypic traits, such as different gender, age, body mass index (BMI), and different stages of type 2 diabetes development. Furthermore, we included intervention studies investigating the effect of caloric restriction or resveratrol ingestion on SAT cell type composition. The results of these analyses are shown in Fig. 6 and in more detail in Supplementary Fig. S10 and Supplementary Data S5.

We detected significant differences in SAT cell composition between males and females, indicating that there are higher amounts of plasmacytoid dendritic cells, $\mathrm{CD} 4^{+} \mathrm{T}$ cells, platelets, and chondrocytes in females, whereas males have more $\mathrm{CD}^{+} \mathrm{T}$ cells, fibroblasts, and endothelial cells. Notably, the differences in $\mathrm{CD} 4^{+}$and $\mathrm{CD} 8^{+} \mathrm{T}$ cells were significant also after correction for multiple testing but were not detected based on single established or CellMaDe-derived markers. With respect to age, we only detected one significant result, indicating a higher amount of fibroblasts with increasing age. 


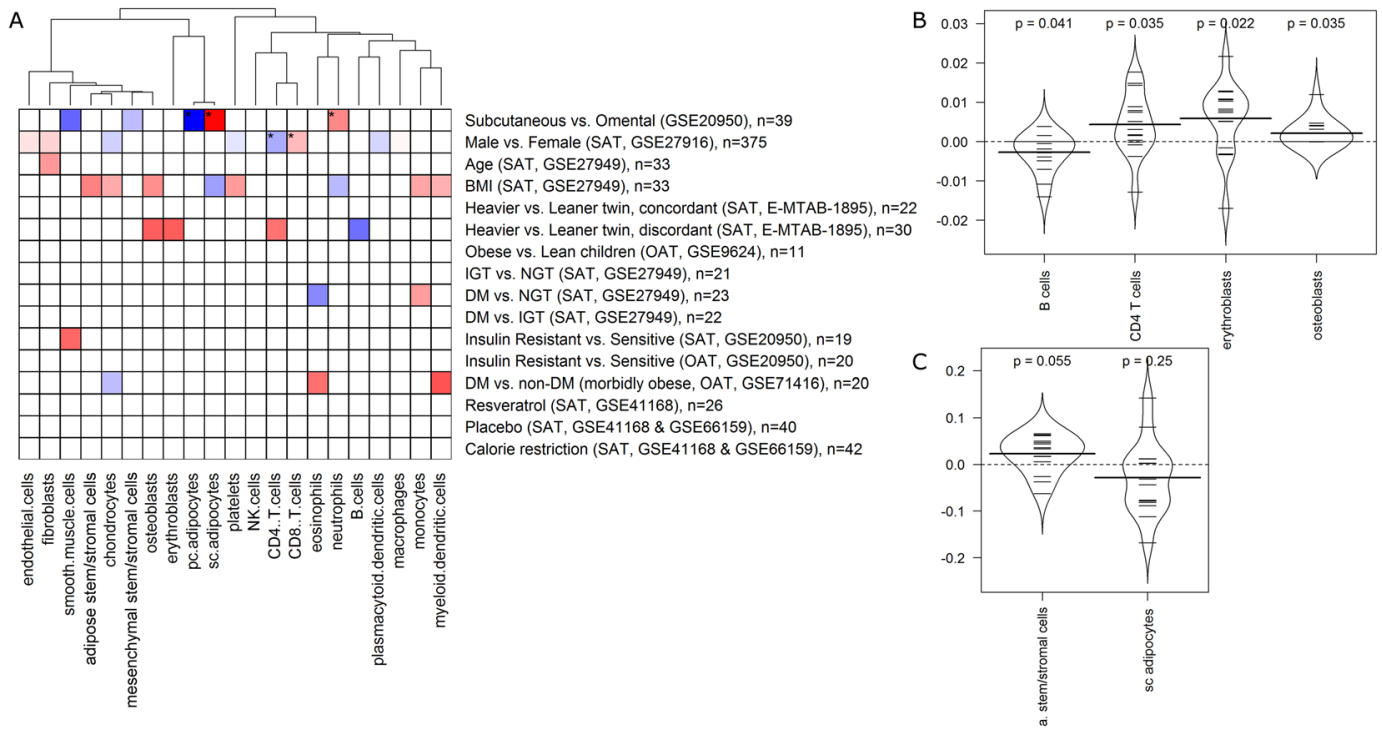

Figure 6. Adipose Tissue Composition Across Phenotypic Traits: (A) Heatmap showing the significant over (red)/under (blue) represented cell type across different categories based on the z-score (denotes the number of standard deviations each group is away from the other). Only significant results (uncorrected $\mathrm{p}<0.05$ ) are colored. Stars label results that stay significant after correction for multiple testing. The bean plots showing (B) all significant cell types and (C) ASCs and subcutaneous adipocytes (not detected as significant), for the heavier vs leaner twin, discordant study. The y axis describes differences in estimated cell fractions between the heavier and leaner twin within a twin pair.

We include four comparisons related to BMI, namely (i) a continuous relationship of BMI with cell fractions in SAT, (ii) a comparison of SAT cellular composition in concordant monozygotic twins (heavier vs. leaner twin, $\Delta \mathrm{BMI}<3 \mathrm{~kg} / \mathrm{m}^{2}$ ), (iii) the same in discordant monozygotic twins (heavier vs. leaner twin, $\Delta \mathrm{BMI}>3 \mathrm{~kg} / \mathrm{m}^{2}$ ), and (iv) a comparison of OAT cellular composition in obese vs. lean children. The first comparison indicates that BMI is positively correlated with the amount of monocytes, myeloid dendritic cells, platelets, osteoblasts, chondrocytes, and ASCs in SAT, whereas the correlation with subcutaneous adipocytes is negative. No significant differences are detected between concordant twins, whereas discordant twins differ significantly in the amount of $\mathrm{CD}^{+} \mathrm{T}$ cells, erythroblasts, osteoblasts (all higher in heavier twins), as well as B cells (higher in leaner twins) (Fig. 6B). Notably, there is a tendency towards higher amounts of ASCs and lower amounts of adipocytes in heavier twins (Fig. 6C), supporting the respective significant finding in the continuous association with BMI. In contrast, some other findings of the continuous association with BMI are not detected in the twin study, which can be attributed to potential confounding effects (age, gender, or other factors) in the continuous association.

The lack of any significant results in the comparison of lean vs. obese children is mostly due to limited power since the study only involves a total of 11 samples ( 5 obese and 6 lean children).

We made six comparisons related to diabetes, glucose tolerance, or insulin resistance. Three of them (impaired glucose tolerance vs. normal glucose tolerance in SAT; diabetes mellitus vs. impaired glucose tolerance in SAT; and insulin-resistant vs. sensitive in OAT) did not show any statistically significant results. The comparison of SAT cell type composition in insulin-resistant vs. sensitive individuals revealed a significant increase in smooth muscle cells in insulin resistant individuals. The SAT of people with diabetes mellitus contains more monocytes and fewer eosinophils than that of normal glucose tolerant people, according to our analysis, whereas the OAT of morbidly obese people shows differences in myeloid dendritic cells, eosinophils (both higher in people with diabetes), and chondrocytes (higher in people without diabetes).

We did not find any evidence that caloric restriction or resveratrol supplementation significantly changes adipose tissue cell type composition.

Interestingly, one included study also reports macrophage percentages as determined by immunohistochemistry $^{25}$ (GSE20950). They show macrophage frequencies for 11 OAT samples (out of 20 study participants) from insulin sensitive and insulin resistant people, with an average macrophage content of $1.8 \%$ (after unit conversion to $\%$ of total cells), which nicely matches our estimate of $1.495 \%$ as average of all 20 participants (selection criteria of the 11 samples for immunohistochemistry were not included in the study description). They furthermore report a significant association between HOMA-IR and macrophage content in these 11 people, which we partially confirm with borderline significance ( $p$-value of 0.054 ) for a comparison of insulin-resistant versus insulin sensitive people in all 20 study participants.

\section{Discussion}

Tissues consist of numerous different cell types and this heterogeneity plays a prominent role in tissue homeostasis in health and disease. In this work, we propose the cell type composition of a tissue, specifically adipose tissue, as a way to investigate the differences across individuals as well as biological conditions. 
Here we describe our novel findings on the cell type composition of the human adipose tissue. We compare our findings to the existing body of knowledge attained via a range of methods (immunohistochemistry, flow cytometry, microbead isolation and enzymatic isolation) by including a review of the studies addressing quantification of cell types (macrophages, $\mathrm{CD}^{+} \mathrm{T}$ cells, $\mathrm{CD}^{+} \mathrm{T}$ cells, endothelial cells and ASCs) within the subcutaneous and omental adipose tissue.

The meta-analysis of these studies indicates that, for adipocytes, ASCs and macrophages there is no consensus on the extent of their presence within the adipose tissue. For adipocytes estimates vary from $15 \%$ to over $90 \%{ }^{14-17}$. Our analysis showed that, independent of the technology (both microarray platforms and RNA-Seq) and reference datasets (AT21 and ex-vivo reference), the median fraction of adipocytes are estimated to be in the range of $55 \%$ to $80 \%$ which was recently independently confirmed by Glastonbury and colleagues ${ }^{18}$. It is postulated that macrophages constitute a specific population of cells that can differ greatly due to inter-individual differences. The extent of tissue specificity for macrophages is reviewed elsewhere ${ }^{36}$.

ASCs are considered to be highly suitable for a range of applications in regenerative therapies ${ }^{37-39}$. Despite all the prospects, our understanding of the ASC within the adipose tissue is currently quite limited. The estimates of ASCs within the adipose tissue are varying from 1-27\% of the total cells (Fig. 4B) depending on the exact markers used to define the ASC phenotype as well as the type of method used for isolating ASCs. In this study we robustly define the ASC signature based on twenty one samples from three independent datasets and report that the ASC composition within the adipose tissue can greatly vary from $3 \%$ to over $30 \%$ across individuals with the mean of $14.8 \%$ in SAT (IQR: $12-17.2 \%$ ) and $15.7 \%$ in OAT (IQR: $11.5-18.6 \%$ ). This relatively large variation is potentially due to the dynamic nature of the stem/stromal cell compartment as well as the broad spectrum of individuals selected for this study. These results highlight adipose tissue as the richest source of multipotent stem/stromal cells, outnumbering possibly any other source in the body.

Moreover, we report for the first time gender-specific differences in the immune system compartment within the subcutaneous adipose tissue, with clear enrichment of CD $4^{+} \mathrm{T}$ cells in females and enrichment of CD8 ${ }^{+} \mathrm{T}$ cells in males. This finding is in line with the established notion of higher numbers of CD4 ${ }^{+} \mathrm{T}$ cells and $\mathrm{CD} 4^{+} / \mathrm{CD} 8^{+}$ $\mathrm{T}$ cell ratios in females versus males observed from the peripheral blood mononuclear cell (PBMC) compartment. This gender difference is reported to remain constant from birth to old age and has potential consequences for a range of conditions including susceptibility to infectious diseases as well as risk for autoimmunity and non-reproductive cancers ${ }^{40}$. The role of gender-specific differences in adipose tissue $\mathrm{CD} 4^{+} / \mathrm{CD} 8^{+} \mathrm{T}$ cell ratios is yet to be further investigated.

We also compared the cell type composition of different adipose tissue depots, revealing high stem/stromal cell percentages (12.4\%-17.2\%) in all depots, identifying SAT as richest in adipocytes (74\%) and EAT and PAT as highly enriched in immune cells, $20.8 \%$, and $20.9 \%$ respectively. Our results implicate robust and systematic differences in cell type composition across adipose tissue depots adding to previously identified differences in metabolism and adipocyte size between depots ${ }^{41}$.

The results highlighted above demonstrate i) cell type composition differences across adipose tissue depots, revealing adipose tissue heterogeneity and assisting future studies investigating the role of a specific adipose tissue depot with a disease phenotype; ii) gender-specific differences in $\mathrm{CD} 4^{+} / \mathrm{CD} 8^{+} \mathrm{T}$ cell ratios within the subcutaneous adipose tissue providing a completely new angle and a potential confounding factor while studying metabolic syndrome; iii) adipose tissue as the richest source of multipotent stem/stromal cells underlining the significance of adipose tissue in the stem cell field, paving the way for future regenerative therapies. Taken together, they demonstrate the power of the TissueDecoder framework in enumeration of cell types within adipose tissue and give an overview of its potential in relating to other fields.

Our approach has various advantages including its ability to analyze many cell types in parallel, its relatively high level of standardization, and its compatibility with retrospective datasets, allowing for meta-analyses of public data from various laboratories. Furthermore, it has the potential of being platform (e.g. Affymetrix or Illumina microarrays) and technique (microarray or RNA-Seq) independent, although most accurate results are currently produced by using the same platform for the reference dataset and analysis dataset, as employed in our study.

Compared to the recently published study by Glastonbury et al. ${ }^{18}$, who followed a similar approach, we include a much broader range of cell types in our signature matrix (21 vs. 4), and apply it to a broader range of adipose tissue depots (SAT, OAT, PAT, EAT), measurement techniques (microarray and RNA-Seq) and original studies.

Naturally, the methodology proposed also comes with shortcomings that can be addressed in future versions or by other studies. One potential extension would be the inclusion of brown/beige adipocytes in the reference dataset in conjunction with an inclusion of adipose tissue depots harboring brown adipose tissue such as supraclavicular, paravertebral, mediastinal, para-aortic and suprarenal depots. Another possibility is to adjust the signature matrix to be suitable for deconvolution of other tissues, as we recently did for deconvolution of skin samples $^{42}$.

Furthermore, generating a reference dataset in which all cell types are isolated from the adipose tissue from healthy individuals covering both age and gender spectrum, could potentially be better for the accuracy of the method since for instance macrophages are known to have distinct expression depending on their tissue of ori$\operatorname{gin}^{36}$. Although still under heated discussion which cell sub-populations reported by single cell transcriptomics reports should be classified as novel cell types, we propose to use the reports from these analyses when there is consensus in the field to identify the cell types for generation of the reference sets ${ }^{43,44}$. Arguably, an optimized reference dataset would also improve the proposed CellMaDe approach for detection of potential novel cell-type specific primary markers.

Finally, in order to study the relation of phenotypic traits with adipose tissue cell type composition in more detail, it is necessary to include studies with larger sample sizes, possibly a few hundred samples, which in our case was only available for the comparison of gender differences. 


\section{Conclusion}

In conclusion, we report a novel methodology to quantify the tissue cell type composition in adipose tissue that can be used both in future studies as well as in existing datasets employing gene expression quantification with our established signature matrix. In generalized terms, our framework allows tissue cell type composition to emerge as a potential marker measuring homeostasis that can be utilized in prospective studies in regenerative medicine. The overview of studies presented in comparison with our findings, reflect the state of the adipose tissue field from the lens of tissue cell type composition. Finally, reported novel cell type-specific markers have great potential in identifying difficult cell types (such as adipose tissue macrophages and eosinophils) and are promising candidates to be evaluated in future studies working with adipose tissue samples.

\section{Data availability}

All data generated during this study and references to the used publicly available data, are included in this published article [and its supplementary information Files].

Received: 11 January 2020; Accepted: 21 May 2020;

Published online: 26 June 2020

\section{References}

1. Vegiopoulos, A., Rohm, M. \& Herzig, S. Adipose tissue: between the extremes. EMBO J. 36, 1999-2017 (2017).

2. Parlee, S. D., Lentz, S. I., Mori, H. \& MacDougald, O. A. Quantifying Size and Number of Adipocytes in Adipose Tissue. Methods Enzymol 537, 93-122 (2014).

3. Kloting, N. \& Bluher, M. Adipocyte dysfunction, inflammation and metabolic syndrome. Rev. Endocr. Metab. Disord. 15, 277-287 (2014).

4. Mraz, M. \& Haluzik, M. The role of adipose tissue immune cells in obesity and low-grade inflammation. J Endocrinol 222, R113-R127 (2014).

5. Newman, A. M. et al. Robust enumeration of cell subsets from tissue expression profiles. Nat. Meth 12, 453-457 (2015).

6. Bourin, P. et al. Stromal cells from the adipose tissue-derived stromal vascular fraction and culture expanded adipose tissue-derived stromal/stem cells: a joint statement of the international federation for adipose therapeutics and science (IFATS) and the international society for cellular therapy (ISCT). Cytotherapy 15(6), 641-648 (2013).

7. Edgar, R., Domrachev, M. \& Lash, A. E. Gene Expression Omnibus: NCBI gene expression and hybridization array data repository. Nucleic Acids Res 30, 207-210 (2002).

8. Dominici, M. et al. Minimal criteria for defining multipotent mesenchymal stromal cells. The International Society for Cellular Therapy position statement. Cytotherapy 8, 315-317 (2006).

9. Gene Ontology Consortium. Gene Ontology Consortium: going forward. Nucleic Acids Res 43, D1049-D1056 (2015).

10. Ferenbach, D. \& Hughes, J. Macrophages and dendritic cells: what is the difference? Kidney Int. 74, 5-7 (2008).

11. Gottfried, E. et al. Expression of CD68 in Non-Myeloid Cell Types. Scand. J. Immunol. 67, 453-463 (2008).

12. Chistiakov, D. A., Killingsworth, M. C., Myasoedova, V. A., Orekhov, A. N. \& Bobryshev, Y. V. CD68/macrosialin: not just a histochemical marker. Lab. Invest 97, 4-13 (2017).

13. Yagi, M. et al. Induction of DC-STAMP by Alternative Activation and Downstream Signaling Mechanisms. J. Bone Miner. Res. 22, 992-1001 (2007).

14. Rink, J. D., Simpson, E. R., Barnard, J. J. \& Bulun, S. E. Cellular characterization of adipose tissue from various body sites of women. J. Clin. Endocrinol. Metab. 81, 2443-2447 (1996).

15. van Harmelen, V., Skurk, T. \& Hauner, H. Primary culture and differentiation of human adipocyte precursor cells. Methods Mol. Med. 107, 125-135 (2005).

16. Hauner, H. Secretory factors from human adipose tissue and their functional role. Proc. Nutr. Soc. 64, 163-169 (2005).

17. Eto, H. et al. Characterization of structure and cellular components of aspirated and excised adipose tissue. Plast. Reconstr. Surg. 124, 1087-1097 (2008).

18. Glastonbury, C. A., Couto Alves, A., El-Sayed Moustafa, J. S. \& Small, K. S. Cell-type heterogeneity in adipose tissue is associated with complex traits and reveals disease-relevant cell-specific eQTLs. Am. J. Hum. Genet. 104, 1013-1024 (2019).

19. Yoshimura, K. et al. Characterization of freshly isolated and cultured cells derived from the fatty and fluid portions of liposuction aspirates. J. Cell. Physiol. 208, 64-76 (2006).

20. Lenz, M. et al. Estimating real cell size distribution from cross-section microscopy imaging. Bioinformatics 32, i396-i404 (2016).

21. Cancello, R. et al. Reduction of Macrophage Infiltration and Chemoattractant Gene Expression Changes in White Adipose Tissue of Morbidly Obese Subjects After Surgery-Induced Weight Loss. Diabetes 54, 2277-2286 (2005).

22. Harman-Boehm, I. et al. Macrophage Infiltration into Omental Versus Subcutaneous Fat across Different Populations: Effect of Regional Adiposity and the Comorbidities of Obesity. J. Clin. Endocrinol. Metab 92, 2240-2247 (2007).

23. Apovian, C. M. et al. Adipose Macrophage Infiltration Is Associated With Insulin Resistance and Vascular Endothelial Dysfunction in Obese Subjects. Arterioscler. Thromb. Vasc. Biol. 28, 1654-1659 (2008).

24. Aron-Wisnewsky, J. et al. Human Adipose Tissue Macrophages: M1 and M2 Cell Surface Markers in Subcutaneous and Omental Depots and after Weight Loss. J. Clin. Endocrinol. Metab. 94, 4619-4623 (2009).

25. Hardy, O. T. et al. BMI-independent inflammation in omental adipose tissue associated with insulin resistance in morbid obesity. Surg. Obes. Relat. Dis. 7, 60-67 (2011).

26. Michaud, A., Drolet, R., Noël, S., Paris, G. \& Tchernof, A. Visceral fat accumulation is an indicator of adipose tissue macrophage infiltration in women. Metabolism 61, 689-698 (2012).

27. Zimmerlin, L. et al. Stromal vascular progenitors in adult human adipose tissue. Cytometry A 77, 22-30 (2010).

28. Aronowitz, J. A., Lockhart, R. A. \& Hakakian, C. S. Mechanical versus enzymatic isolation of stromal vascular fraction cells from adipose tissue. Springerplus 4, 713 (2015).

29. Aronowitz, J. A. \& Ellenhorn, J. D. Adipose stromal vascular fraction isolation: a head-to-head comparison of four commercial cell separation systems. Plast. Reconstr. Surg. 132, 932e-939e (2013).

30. Viardot, A., Lord, R. V. \& Samaras, K. The Effects of Weight Loss and Gastric Banding on the Innate and Adaptive Immune System in Type 2 Diabetes and Prediabetes. J. Clin. Endocrinol. Metab 95, 2845-2850 (2010).

31. Ehrlund, A. et al. The cell-type specific transcriptome in human adipose tissue and influence of obesity on adipocyte progenitors. Sci. Data 4, 170164 (2017).

32. Ojha, S. et al. Gene pathway development in human epicardial adipose tissue during early life. JCI Insight 1, e87460 (2016).

33. Hägg, S. et al. Multi-organ expression profiling uncovers a gene module in coronary artery disease involving transendothelial migration of leukocytes and LIM domain binding 2: the Stockholm Atherosclerosis Gene Expression (STAGE) study. PloS Genet 5, e1000754 (2009).

34. Hirata, Y. et al. Enhanced inflammation in epicardial fat in patients with coronary artery disease. Int Heart J 52, 139-142 (2011) 
35. Mazurek, T. et al. Human Epicardial Adipose Tissue Is a Source of Inflammatory Mediators. Circulation 108, 2460-2466 (2003).

36. Davies, L. C. \& Taylor, P. R. Tissue-resident macrophages: then and now. Immunology 144, 541-548 (2015).

37. Frese, L., Dijkman, P. E. \& Hoerstrup, S. P. Adipose Tissue-Derived Stem Cells in Regenerative Medicine. Transfus. Med. Hemother. 43, 268-274 (2016).

38. De Jaime-Soguero, A. et al. Wnt/Tcf1 pathway restricts embryonic stem cell cycle through activation of the Ink4/Arf locus. PLoS Genet. 13, e1006682 (2017).

39. Bunk, E. C. et al. Prox1 Is Required for Oligodendrocyte Cell Identity in Adult Neural Stem Cells of the Subventricular Zone. Stem Cells 34, 2115-2129 (2016).

40. Klein, S. L. \& Flanagan, K. L. Sex differences in immune responses. Nat. Rev. Immunol. 16, 626-638 (2016)

41. Bjørndal, B., Burri, L., Staalesen, V., Skorve, J. \& Berge, R. K. Different Adipose Depots: Their Role in the Development of Metabolic Syndrome and Mitochondrial Response to Hypolipidemic Agents. J. Obes 2011, 490650 (2011).

42. Felix Garza, Z. C. et al. Characterization of disease-specific cellular abundance profiles of chronic inflammatory skin conditions from deconvolution of biopsy samples. BMC Med Genomics 12, 121 (2019).

43. Rozenblatt-Rosen, O., Stubbington, M. J. T., Regev, A. \& Teichmann, S. A. The Human Cell Atlas: from vision to reality. Nature 550, 451-453 (2017).

44. Acosta, J. R. et al. Single cell transcriptomics suggest that human adipocyte progenitor cells constitute a homogeneous cell population. Stem Cell Res. Ther 8, 250 (2017).

\section{Acknowledgements}

This research has been made possible with support of the Dutch Province of Limburg to the Maastricht Centre for Systems Biology (MaCSBio).

\section{Author contributions}

M.L. and G.E. conceived the project, performed the analyses and wrote the manuscript (all in equal contribution). I.A., R.P. and T.K. were involved in interpretation of results and editing of the manuscript.

\section{Competing interests}

The authors declare no competing interests.

\section{Additional information}

Supplementary information is available for this paper at https://doi.org/10.1038/s41598-020-67177-1.

Correspondence and requests for materials should be addressed to M.L. or G.E.

Reprints and permissions information is available at www.nature.com/reprints.

Publisher's note Springer Nature remains neutral with regard to jurisdictional claims in published maps and institutional affiliations.

Open Access This article is licensed under a Creative Commons Attribution 4.0 International License, which permits use, sharing, adaptation, distribution and reproduction in any medium or format, as long as you give appropriate credit to the original author(s) and the source, provide a link to the Creative Commons license, and indicate if changes were made. The images or other third party material in this article are included in the article's Creative Commons license, unless indicated otherwise in a credit line to the material. If material is not included in the article's Creative Commons license and your intended use is not permitted by statutory regulation or exceeds the permitted use, you will need to obtain permission directly from the copyright holder. To view a copy of this license, visit http://creativecommons.org/licenses/by/4.0/.

(C) The Author(s) 2020 